\title{
Social work theory and methods: The essentials
}

\author{
Neil Thompson and Paul Stepney (Eds.) \\ Routledge, New York, 2018 \\ ISBN 978-1-138-62978-3, pp.251, paperback, NZD67.00
}

S ocial Work Theory and Methods: The Essentials, edited by Neil Thompson and Paul Stepney, aims to be an introduction into the study of social work theory and methods. The editors outline three goals in collating this book: To clarify the difference between theory and method, to highlight the importance of evidence based social work practice, and to offer a 'gateway' to the significant literature base relating to theory and methods. The book is divided into two parts: 'Part 1 - Social Work Theory and Methods in Context: Introduction', which looks at theory and methods in a practice, intellectual and policy and organisational context. 'Part 2 - Social Work Theories and Methods in Practice: Introduction' then outlines 14 different theories and models that inform social work practice, with each chapter written by a different contributor.

This book starts with a useful discussion by Neil Thompson on what is meant by 'theory' and 'method' and introduces Thompson's model of 'theorizing practice' in which he demonstrates how to start with a practice example and then draw on the practitioner's knowledge base in order to formulate a response. I found this chapter to be particularly useful, and I know it would have been very helpful for me as a student and new social worker. It is always important to reflect on your methods of practice, and Thompson here stresses the necessity of having an informed knowledge base, rather than being unduly influenced by assumption and stereotype.

Chapters 2 and 3 go on to explore the importance of 'research informed and evidence enriched practice' and how policy and organisational culture can impact on our ability to engage in critically reflective practice. Particularly useful here was the highlighting of how outcome-based operational models can have a negative impact on the quality of supervision that practitioners receive, with a focus on outcomes rather than reflecting on why certain interventions are used - or not used. A timely reminder to not lose focus on the 'why' of our social work practice.

The book then explores the 14 different theories and models mentioned earlier, ranging from Psychodynamic Theory and Cognitive Behavioural Therapy, all the way through to Family Therapy and Ecological theories. Each chapter provides a concise explanation of the origins of each theory and model, and then outlines how it can be applied to social work practice. Each chapter uses 'Practice Focus' examples to illustrate the use of each theory in a practice situation. This is a useful addition and serves to give the reader an idea of how these theories and models may be applied in the real world. Each chapter also finishes with 'Points to Ponder' - questions to encourage further self-reflection. I can see that these would be useful in supervision, both with students and with more experienced practitioners.

'Chapter 10: Solution-focused practice', written by Suki Desai, looks at the origins of solution-focused practice in family therapy intervention. The writer explains that solution-focused practice is based on the belief that people already possess the skills and resources to solve their own problems, 
and that it is the role of the practitioner to facilitate this process. This model of working gives a more balanced power relationship between the client and the practitioner, and sees the client as being the expert in their situation. The chapter then uses an in-depth practice example to demonstrate how solution-focused practice can be used in a real-life setting. This case example is a social worker working in Community Mental Health who is supporting a 66-year-old woman who has been experiencing severe anxiety and depression. The example follows the social worker through her assessment of the client's situation, and her use of various questioning techniques to establish what the client's view of the situation is and what they would like to gain from their interaction with the social worker. The chapter ends with highlighting the importance of the practitioner taking time to reflect on their approach, and continues to explore how using such an approach can assist the practitioner in the case example in their ongoing work with their client. The use of an in-depth case study, along with the examples of various techniques such as 'scaling' and the use of the 'miracle question', give a helpful starting point for a practitioner wishing to incorporate this approach into their practice.

Overall this book is a good introduction to the importance of theory and models to social work practice, and gives a good outline of many of the more popular methods of practice. It will be of particular value to students who are beginning to build up their knowledge, and is also of use as a refresher to those of us who are more experienced. Social Work Theory and Methods: The Essentials provides a useful starting point for more in-depth study into social work theory and methods.

I found the book to be well written and easy to read. It is, however, a very Western text, with most contributors coming from the United Kingdom or Scandinavia. I feel that this book would have been strengthened with the inclusion of some theory and methods of practice stemming from other cultures. In light of this I would recommend that this book is read alongside other literature that explores social work from an Aotearoa New Zealand perspective. 